\title{
RESEARCH
}

Open Access

\section{The CD200/CD200R signaling pathway contributes to spontaneous functional recovery by enhancing synaptic plasticity after stroke}

Hao Sun, Xinran He, Xia Tao, Tingting Hou, Mingming Chen, Meijun He and Hong Liao * (D)

\begin{abstract}
Background: Spontaneous functional recovery occurs during the acute phase after stroke onset, but this intrinsic recovery remains limited. Therefore, exploring the mechanism underlying spontaneous recovery and identifying potential strategies to promote functional rehabilitation after stroke are very important. The CD200/CD200R signaling pathway plays an important role in neurological recovery by modulating synaptic plasticity during multiple brain disorders. However, the effect and mechanism of action of the CD200/CD200R pathway in spontaneous functional recovery after stroke are unclear.
\end{abstract}

Methods: In this study, we used a transient middle cerebral artery occlusion (MCAO) model in rats to investigate the function of CD200/CD200R signaling in spontaneous functional recovery after stroke. We performed a battery of behavioral tests (Longa test, adhesive removal test, limb-use asymmetry test, and the modified grip-traction test) to evaluate sensorimotor function after intracerebroventricular (i.c.v.) injection with CD200 fusion protein (CD200Fc) or CD200R blocking antibody (CD200R Ab) post-stroke. Density and morphology of dendritic spines were analyzed by Golgi staining. Microglia activation was evaluated by immunofluorescence staining. Western blot was used to detect the levels of protein and the levels of mRNA were measured by qPCR.

Results: Our study demonstrated that sensorimotor function, synaptic proteins, and structures were gradually recovered and CD200R was transiently upregulated in ipsilateral cortex after stroke. Synapse-related proteins and dendritic spines were preserved, accompanied by sensorimotor functional recovery, after stereotaxic CD200Fc injection post-stroke. In addition, CD200Fc restrained microglia activation and pro-inflammatory factor release (such as II-1, Tnf- $a$, and II-6) after MCAO. On the contrary, CD200R Ab aggravated sensory function recovery in adhesive removal test and further promoted microglia activation and pro-inflammatory factor release (such as $11-1$ ) after MCAO. The immune-modulatory effect of CD200/CD200R signaling might be exerted partly by its inhibition of the MAPK pathway.

Conclusions: This study provides evidence that the CD200/CD200R signaling pathway contributes to spontaneous functional recovery by enhancing synaptic plasticity via inhibition of microglia activation and inflammatory factor release.

Keywords: CD200/CD200R, Stroke, Inflammation, Spontaneous recovery, Synaptic plasticity

\footnotetext{
* Correspondence: hliao@cpu.edu.cn

Jiangsu Key laboratory of Drug Screening, China Pharmaceutical University, 24 Tongjiaxiang Street, Nanjing 210009, China
}

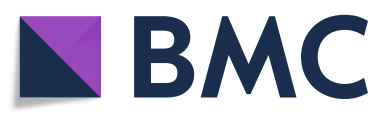

C The Author(s). 2020 Open Access This article is licensed under a Creative Commons Attribution 4.0 International License, which permits use, sharing, adaptation, distribution and reproduction in any medium or format, as long as you give appropriate credit to the original author(s) and the source, provide a link to the Creative Commons licence, and indicate if changes were made. The images or other third party material in this article are included in the article's Creative Commons licence, unless indicated otherwise in a credit line to the material. If material is not included in the article's Creative Commons licence and your intended use is not permitted by statutory regulation or exceeds the permitted use, you will need to obtain permission directly from the copyright holder. To view a copy of this licence, visit http://creativecommons.org/licenses/by/4.0/ The Creative Commons Public Domain Dedication waiver (http://creativecommons.org/publicdomain/zero/1.0/) applies to the data made available in this article, unless otherwise stated in a credit line to the data. 


\section{Introduction}

Stroke, among which ischemic stroke accounts for nearly $87 \%$, is a leading cause of death and disability worldwide, causing $\sim 80 \%$ of all disabilities [1]. Due to improved surgical interventions, an increasing number of patients survive the devastating initial event but suffer from ongoing sensorimotor and cognitive dysfunction [2, 3]. Despite immediate and longlasting spontaneous functional recovery, the degree of recovery remains limited and varies among individuals [3]. Spontaneous recovery involves a variety of cellular and molecular processes, and many restorative therapies depend on the processes observed during spontaneous recovery [4]. Therefore, it is important to investigate the mechanisms underlying spontaneous recovery and identify rehabilitative therapies to augment sensorimotor functional recovery. Such investigations will also improve our understanding of the pathological development of stroke.

The integrity of neuronal networks is the basis of sensorimotor behavior; however, neuronal networks and synapse circuits are damaged by stroke in regions that receive reduced blood supply, which results in sensorimotor dysfunction [5]. The surviving neural networks are partially rewired, and intact synapses are recruited during spontaneous recovery [6]. Thus, replacing the destroyed circuit and promoting surviving neural networks to remap might be treatment strategies to improve functional recovery after stroke.

Inflammation plays an important role in regulating synaptic plasticity [7-9]. Activated microglia are characterized as proinflammatory or anti-inflammatory microglia; microglia activation occurs within hours after stroke, and several pro-inflammatory factors (such as $\mathrm{Il}$ 1, Tnf- $\alpha$, and Il-6) can be released by microglia in response to synapse degeneration or loss $[10,11]$. Thus, inhibiting microglia activation and pro-inflammatory factor release may enhance synaptic plasticity and promote long-term stroke recovery. The CD200/CD200R signaling pathway has a unique expression profile, and it plays a profound regulatory role during neurological recovery under multiple brain pathological conditions. CD200, a member of the immunoglobulin superfamily, is widely expressed in neurons, astrocytes, and oligodendrocytes, whereas its receptor (CD200 receptor 1, CD200R1) is expressed in myeloid cells and microglia (the brain-resident myeloid cells) in rodents [12] and also highly expressed by neurons in human according to the Ben Barres database. The interaction between CD200 and CD200R1 is critical for inhibiting microglial activation and localized neuroinflammation during the pathological development of several brain diseases, including Parkinson's disease, optic nerve crush, and germinal matrix hemorrhage [13-15]. The CD200/CD200R signaling pathway also participates in synaptic plasticity. Suppressing CD200/CD200R signaling via genetic approaches (deleting $\mathrm{Cd} 200$ ) impairs long-term potentiation (LTP) [16], whereas pharmaceutical approaches (using CD200Fc, a CD200R agonist) that activate CD200/CD200R signaling significantly enhance synaptic plasticity in $\mathrm{AD}$ and aged mice by regulating inflammation [17-19]. However, the role of the CD200/CD200R signaling pathway during spontaneous functional recovery remains unclear.

Here, we propose that the CD200/CD200R signaling pathway contributes to spontaneous functional recovery after stroke by restraining synapse loss through inhibiting microglia activation and pro-inflammatory factor release.

\section{Methods \\ Animals}

Adult male Sprague-Dawley (SD) rats (280-320 g, 810 weeks of age) were purchased from Shanghai Sippr-BK laboratory animal Co. Ltd. (Shanghai, China) and housed in the Pharmaceutical animal experimental center of China Pharmaceutical University under a 12 light-dark cycle with food and water ad libitum. All animal procedures were approved by the Animal Research Ethics Committee of China Pharmaceutical University.

\section{Animal surgery and drug administration}

A transient right middle cerebral artery occlusion (MCAO) stroke was performed as previously described [20]. Briefly, rats were anesthetized with $2 \%$ isoflurane, then exposing the right common carotid artery of rats. A 3-0 poly-lysine-coated monofilament nylon suture was inserted into the right internal carotid artery through the external carotid artery stump to occlude the middle cerebral artery. Two hours later, the filament was withdrawn to restore blood flow. Warming pads were used to maintain the body temperatures of animals at $37.0 \pm$ $0.5^{\circ} \mathrm{C}$, and Doppler flowmetry (Moor Instruments, Essex, UK) was used to monitor cerebral blood flow (CBF) during the surgical procedure. In the ischemia phase, cerebral blood flow $<25 \%$ of the baseline was considered as successful ischemia.

The animals were anesthetized with $2 \%$ isoflurane and placed on a stereotaxic apparatus (RWD Life Science, Shenzhen, China). After exposing the skull, a small hole was made to allow the intracerebroventricular injection. CD200Fc $(5 \mu \mathrm{L}, 4 \mu \mathrm{g} / \mu \mathrm{L})$ (Jiangsu Futai Biotechnology, Taizhou, China) as an agonist of CD200R1, antiCD200R1 antibody (CD200R Ab) (AbD Serotec, Oxford, UK) as an antagonist of CD200R1 $(5 \mu \mathrm{L})$, or IgG (Santa Cruz Biotechnology Inc, USA) was injected into the right cerebral ventricle one time by a $10-\mu \mathrm{L}$ Hamilton 
syringe $24 \mathrm{~h}$ after MCAO. The injection was controlled at a rate of $0.5 \mu \mathrm{L} / \mathrm{min}$ and the syringe remained in place for $5 \mathrm{~min}$ before the completion of injection. The stereotaxic coordinates of the right cerebral ventricle were as follows: bregma $-0.8 \mathrm{~mm}$; lateral $-1.5 \mathrm{~mm}$; ventral $4.0 \mathrm{~mm}$.

\section{Behavioral tests}

Behavioral tests were performed at 4, 7, 14, 21, and 28 days after MCAO by an investigator blinded to the experimental groups.

\section{Longa test}

This test was assessed using a 5-point scale as described previously [20]: 0 , no observable deficits; 1 , failure to extend the left forepaw; 2 , circling to the left; 3 , falling to the left; and 4, unable to move spontaneously.

\section{Adhesive removal test}

To measure the sensory functions and motor functions, the tests were performed as described previously [21] with some modifications. The time that rats contacted and removed the spot was recorded and within a limit of $180 \mathrm{~s}$.

\section{Limb-use asymmetry test (cylinder)}

To evaluate forelimb-use asymmetry, rats were placed in a transparent cylinder (diameter $20 \mathrm{~cm}$ and height 45 $\mathrm{cm}$ ). The times of touching the wall by the forelimb of rats were recorded. The detailed operation was described previously [22].

\section{The modified grip-traction test}

Rats were hung to a horizontal rope (a 0.6-cm-diameter plastic tube placed horizontally $45 \mathrm{~cm}$ above the table) by its forepaws to evaluate the muscle strength [23]. Time of fall (maximum $60 \mathrm{~s}$ ) was noted.

\section{Tissue preparation}

Rats were anesthetized with ketamine and xylazine in $0.9 \%$ saline at 4,7 , and 28 days after MCAO and perfused with $0.9 \%$ saline, followed by fresh cold $4 \%$ PFA in $0.9 \%$ saline. Brain tissues were fixed in fresh $4 \%$ PFA $4{ }^{\circ} \mathrm{C}$ overnight and dehydrated gradient in $20 \%$ and $30 \%$ sucrose at $4{ }^{\circ} \mathrm{C}$ before sectioning on a Leica1950 cryostat (Leica Instruments, Germany) at $10 \mu \mathrm{m}$ and $30 \mu \mathrm{m}$. Sections were stored at $-20^{\circ} \mathrm{C}$ for immunohistochemistry.

\section{Cresyl violet staining}

Cerebral infarct volume was detected at 4 days, 7 days, and 28 days after MCAO [24]. Rats were anesthetized and perfused with physiological saline followed by $4 \%$ paraformaldehyde (PFA) at 4 days, 7 days, and 28 days after MCAO. The brains were removed carefully and fixed in PFA for $24 \mathrm{~h}$, then dehydrated in $20 \%$ and $30 \%$ sucrose for $24 \mathrm{~h}$ respectively in $4{ }^{\circ} \mathrm{C}$. The brain tissue was cut serially at $30 \mu \mathrm{m}$ in coronal plan. The sections were stained by cresyl violet.

\section{Immunofluorescence}

Brain sections were permeabilized by $0.3 \%$ Triton- 100 in PBS, blocked by $10 \%$ goat serum in $90 \%$ PBS at room temperature for $1 \mathrm{~h}$, and then incubated primary antibody at $4{ }^{\circ} \mathrm{C}$ overnight. The primary antibodies were as follows: mouse anti-CD200R (1:50, Bio-Rad), mouse anti-CD68 (1:300, Bio-Rad), and anti-Iba1 (1:300, Wako, Japan). After rinsed three times, sections were incubated with Alexa Flour 488 conjugated goat anti-rabbit IgG (1:500, Invitrogen) and Alexa Flour 633 conjugated goat anti-mouse IgG (1:1000, Invitrogen) at room temperature for $1 \mathrm{~h}$. The fluorescent imaging was collected by an Olympus fluorescence microscope and processed by ImageJ software.

\section{Golgi-Cox staining}

Animals were sacrificed and brain tissues were removed at 28 days after MCAO. Samples were immersed in Golgi-Cox solutions for 2 weeks and then dehydrated gradient in $20 \%$ and $30 \%$ sucrose at $4{ }^{\circ} \mathrm{C}$. Tissues were cut into $100-\mu \mathrm{m}$ sections by Leica1950 cryostat (Leica Instruments, Germany) for following staining. Sections were immersed in $75 \%$ ammonia solution and $1 \%$ sodium thiosulfate. Images were collected on a microscope, and 10 20 neurons per sample were randomly observed for morphological analysis.

\section{BV2 cell culture and oxygen-glucose deprivation (OGD)} Cells from the immortalized mouse microglia cell line BV-2 were cultured in DMEM containing $10 \%$ fetal bovine serum (FBS) and maintained at $37^{\circ} \mathrm{C}$ in $5 \%$ $\mathrm{CO}_{2}$ and $95 \%$ oxygen. BV2 were treated with IgG (15 $\mu \mathrm{g} / \mathrm{ml})$ or CD200Fc $(15 \mu \mathrm{g} / \mathrm{ml}) 30 \mathrm{~min}$ before OGD. BV2 were subjected to OGD as previously reported $[25,26]$ with a slight modification. For OGD induction, BV2 culture medium was replaced with OGD buffer that contained $4.09 \mathrm{~g} \mathrm{NaCl}, 186.5 \mathrm{mg} \mathrm{KCl}, 111$ $\mathrm{g} \mathrm{CaCl}_{2}, 5 \mathrm{ml} \mathrm{HEPES}$, and $1.125 \mathrm{~g}$ glycine in a total volume of $500 \mathrm{ml}$. Then, BV2 were kept in a humidified atmosphere containing 95\% nitrogen and 5\% $\mathrm{CO}_{2}$ for $6 \mathrm{~h}$ and reoxygenation for $16 \mathrm{~h}$. For reoxygenation, OGD buffer was replaced with BV2 culture medium and cells were returned to normoxic conditions (95\% air and 5\% CO2). BV2 cultured under the normoxic condition served as the control. 
To determine whether CD200Fc could inhibit JNK and P38 phosphorylation in BV2 cell, CD200Fc $(15 \mu \mathrm{g} / \mathrm{ml})$ or IgG $(15 \mu \mathrm{g} / \mathrm{ml})$ in the presence of anisomycin $(1 \mathrm{nM})$ was added to the BV2 for $30 \mathrm{~min}$, and BV2 cultured under the normoxic condition served as the control.

\section{Real-time quantitative RT-PCR (RT-qPCR)}

At 4,7 , and 28 days after MCAO, brain samples were collected from ipsilateral sensorimotor cortex. Total RNA was isolated from frozen tissues using TRIzol Reagent (Vazyme, Nanjing, China) according to the manufacturer's protocol. Equal amounts of total RNA were reverse transcribed under standard condition using HiScript 1st Strand cDNA Synthesis Kit (Vazyme, Nanjing, China). Quantitative PCR was performed on an ABI7000 real-time PCR system (Applied Biosystems, Inc., University Park, IL, USA) using SYBR Green Master Mix (Vazyme, Nanjing, China). The cycle time values were normalized to GAPDH of the same sample. Primer sequences are shown in Table 1.

\section{Western blot}

At 4, 7, and 28 days after MCAO, brain samples were collected from the ipsilateral sensorimotor cortex. Brain tissues were homogenized with RIPA lysate (Beyotime, Nanjing, China) supplied with protease inhibitor cocktail (Roche, Indianapolis, IN, USA). An equal amount of protein was separated on SDS polyacrylamide gels, transferred to polyvinyl-difluoride membranes (Millipore, Billerica, MA, USA), and blocked with $2.5 \%(\mathrm{w} / \mathrm{v})$ bovine serum albumin (BSA). The membrane was incubated with primary antibody (mouse anti-CD200R, 1:500, Bio-Rad, USA goat antiCD200, 1:2000, RD, USA; rabbit anti-p-P65, 1:1000, CST, USA; rabbit anti-P65, 1:1000, CST, USA; rabbit anti-p-ERK, 1:1000, CST, USA; rabbit anti-ERK, 1: 1000, CST, USA; rabbit anti-p-JNK, 1:1000, CST, USA; rabbit anti-JNK, 1:1000, CST, USA; rabbit antip-P38, 1:1000, CST, USA; rabbit anti-P38, 1:1000, CST, USA; mouse anti-actin, 1:1000, Santa Cruz Biotechnology Inc, USA) at $4{ }^{\circ} \mathrm{C}$ overnight. After being rinsed three times ( $7 \mathrm{~min} /$ time) with TBST, the membrane was incubated corresponding secondary antibody at room temperature for $1 \mathrm{~h}$. After washed, the

Table 1 Primer sequences

\begin{tabular}{|c|c|c|}
\hline Gene & Forward primer $\left(5^{\prime}-3^{\prime}\right)$ & Reverse primer $\left(5^{\prime}-3^{\prime}\right)$ \\
\hline$\| 11 \beta$ & TCCAGGATGAGGACCCAAGC & TCGTCATCATCCCACGAGTCA \\
\hline$\|-6$ & CAGC & AT \\
\hline Tnf-a & TTCCCAAATGGGCTCCCTCT & GTGGGCTACGGGCTTGTCAC \\
\hline$\|-4$ & CAGGGTGCTTCGCAAATTITAC & ACCGAGAACCCCAGACTTGTT \\
\hline Cd206 & GGTTCCGGTTTGTGGAGCAG & TCCGTTTGCATTGCCCAGTA \\
\hline apdh & CAGCCTCGTCTCATAGACAAGATG & AAGGCAGCCCTGGTAACCA \\
\hline
\end{tabular}

labeled proteins were detected using the Bio-Rad system (Bio-Rad, Germany).

\section{Statistical analysis}

The GraphPad Prism Software 6 (La Jolla, CA, USA) was used for statistical analysis, and the results were presented as a mean \pm SEM. Among the frequencies, Longa test (Fig. 1a and Fig. 4a) was analyzed by non-parametric Mann-Whitney test. Adhesive removal test, limb-use asymmetry test (cylinder), and the modified grip-traction test (Fig. 1b-e and Fig. 4b-e) were performed by using two-way analysis of variance (ANOVA) with post hoc Student-Newman-Keuls test. For experiments with more than two groups and two factors of the parameters, results were compared using two-way analysis of variance (ANOVA) followed by post hoc Student-Newman-Keuls test. All tests were considered statistically significant at $P<0.05$.

\section{Results}

Sensorimotor functional spontaneous recovery after stroke in rats

Sensorimotor functions were first assessed by performing a series of behavioral tests after MCAO surgery. Briefly, the Longa test for neurological disorders was performed (Fig. 1a), and sensorimotor dysfunction was identified using adhesive removal, limb-use asymmetry, and grip-traction tests (Fig. 1b-e). MCAO-induced neurological disorder observed at 4,7 , and 14 days improved gradually at 21 and 28 days compared with 4 days after MCAO $(P<0.05)$ (Fig. 1a). In the adhesive removal test, rats spent more time touching the spot at 4,7 , and 14 days after MCAO (time main effect, $F_{4,110}=8.819, P$ $<0.0001$ ) and spent more time removing the spot at 4 , 7,14 , and 21 days after MCAO compared with the sham group (time main effect, $F_{4,110}=4.222, P=0.0032$ ) (Fig. 1b, c). The time taken to touch or remove the spot decreased gradually as spontaneous recovery proceeded compared with 4 days $(t$-test, $P<0.05)$ (Fig. 1b, c). The injured forelimb mobility of MCAO rats was visibly reduced at each time point after MCAO, as determined by the limb-use asymmetry test (time main effect, $F_{4,121}=$ $0.6806, P=0.6067$ ) (Fig. 1d). The hanging time was significantly reduced at $4,7,14,21$, and 28 days in the MCAO group compared with the sham group, and partial recovery occurred at 14,21 , and 28 days after MCAO compared with 4 days $(t$-test, $P<0.05)$ (Fig. $1 \mathrm{~d})$.

When the size of the cerebral infarction was assessed by cresyl violet staining, the size decreased over time $\left(F_{3,24}=101.0, P<0.0001\right)$ (Fig. 1f, g). Taken together, these data suggest that neurological dysfunction occurred immediately after MCAO, followed by slow and limited spontaneous recovery. 


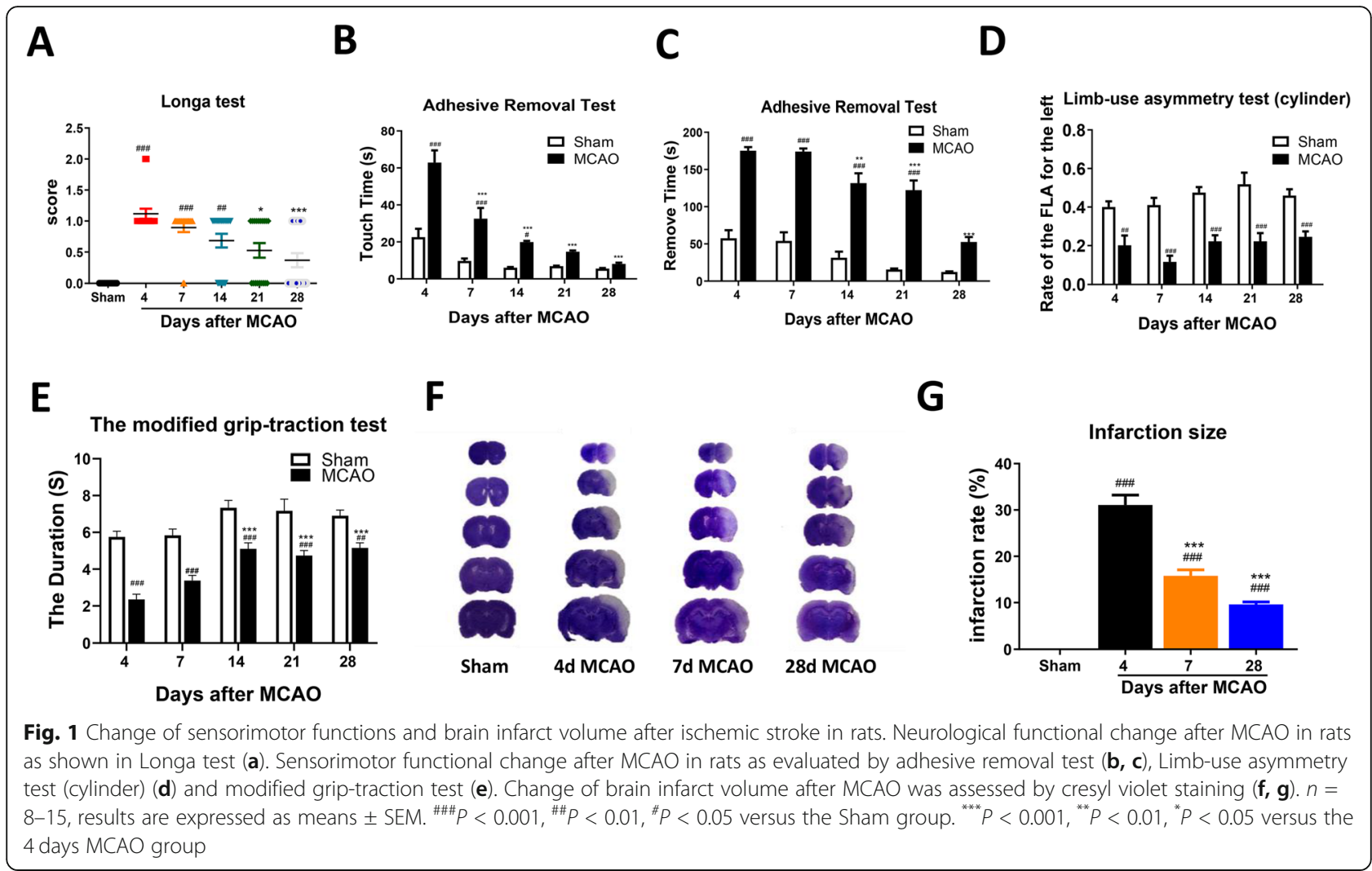

Expression of synapse-related proteins and synaptic loss after cerebral ischemia in rats

Synaptic plasticity plays an important role in functional recovery after stroke [27, 28], and synapserelated proteins and dendritic spine density are important indices of synaptic plasticity. Thus, we measured the protein levels of postsynaptic density protein 95 (PSD-95) and synaptophysin (SYP) in the ipsilateral sensorimotor cortex. The protein levels of $\operatorname{PSD} 95\left(F_{3,8}=17.51, P=0.0007\right)$ and SYP $\left(F_{3,8}\right.$ $=43.22, P<0.0001)$ were decreased significantly at 4,7 , and 28 days after MCAO compared with the sham group (Fig. $2 \mathrm{a}-\mathrm{c}$ ). However, compared with the 4-day MCAO group, the expression of PSD95 was increased markedly at 28 days after MCAO $(t$ test, $P<0.05$ ) (Fig. 2a, b). When dendritic spine density and morphology in the ipsilateral sensorimotor cortex were measured, dendritic spine density was reduced dramatically ( $t$-test, $P<0.0001)$, the percent stubby spine was increased significantly, and the percent mushroom spine was decreased significantly at 28 days after MCAO (group main effect, $F_{2,12}=66.62, P<0.0001$ ) (Fig. $2 \mathrm{~d}-\mathrm{f}$ ). These data suggest the presence of markedly decreased synaptic function after stroke and slow recovery following the initial event.
The expression of CD200 and CD200R after cerebral ischemia in rats

Based on the important role of the CD200/CD200R signaling pathway on regulating synaptic plasticity in many pathological conditions [16, 29, 30], we investigated the expression of CD200 and CD200R in the ipsilateral sensorimotor cortex. There were no obvious differences in the expression of CD200 between the sham and MCAO groups (group main effect, $F_{3,8}=1.468, P=0.2947$ ) (Fig. 3a, b). However, the expression of CD200R was increased markedly at 4 days after MCAO compared with the sham group (group main effect, $F_{3,8}=11.36, P=0.0030$ ) (Fig. 3)c, d. Immunofluorescent staining showed that CD200R was expressed on Iba1-positive cells (Fig. 3e). These results suggest that the CD200/CD200R signaling pathway may be involved in spontaneous functional recovery after stroke onset in rats.

\section{Functional outcomes after CD200/CD200R signaling} pathway modification post-cerebral ischemia in rats To determine whether modulating CD200/CD200R signaling affects spontaneous functional recovery, we performed a battery of behavioral tests to evaluate sensorimotor function after intracerebroventricular (i.c.v.) injection with CD200 fusion protein (CD200Fc) or CD200R 


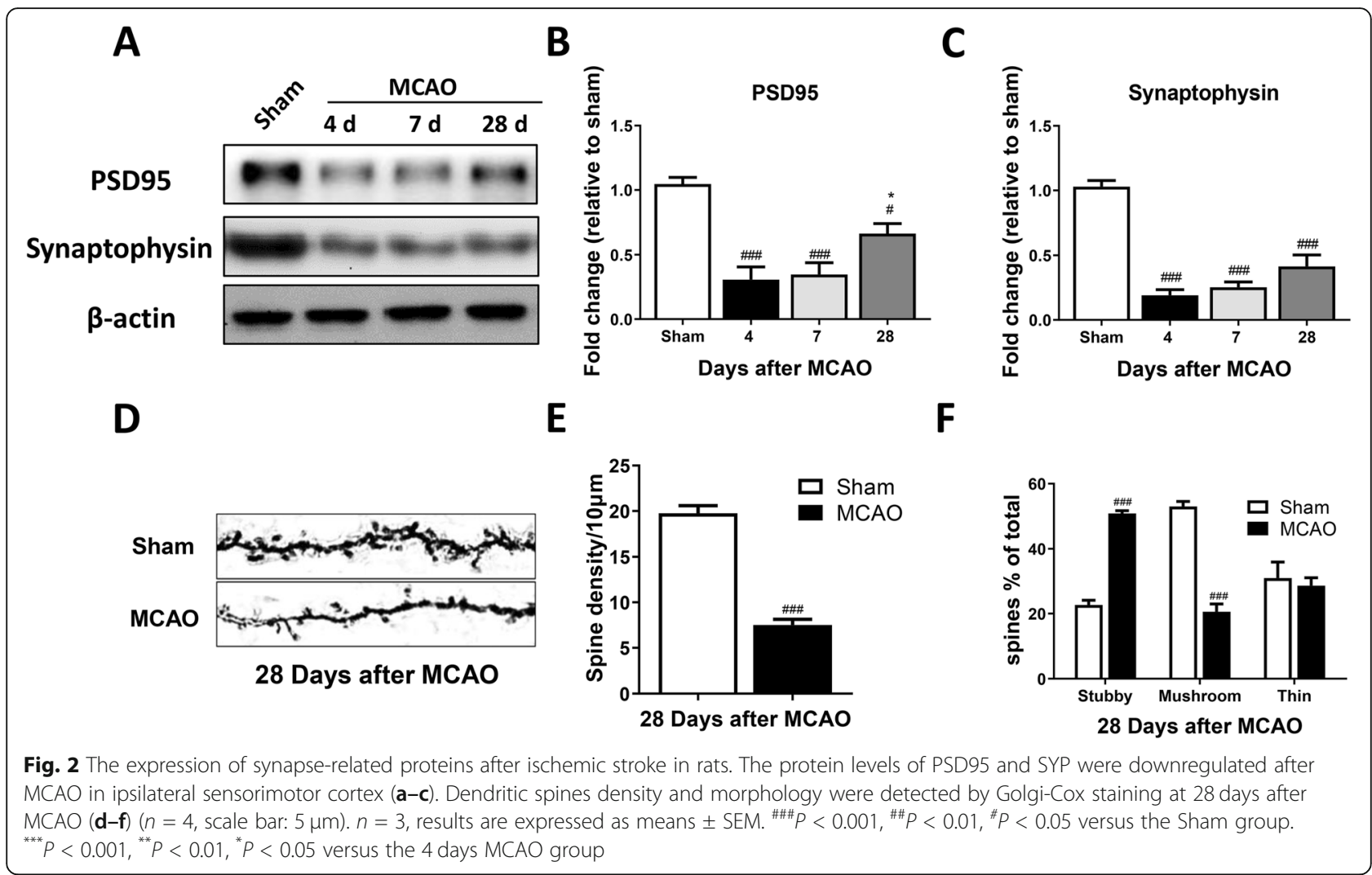

blocking antibody (CD200R Ab) post-stroke. First, CD200Fc significantly improved neurological recovery in Longa tests at 4 and 7 days after MCAO, whereas neurological recovery was restrained at 4 days after CD200R Ab injection compared with the MCAO+IgG group $(P<0.05)$ (Fig. 4a). Second, sensorimotor functions were assessed using adhesive removal, limb-use asymmetry, and modified grip-traction tests. Rats spent more time touching the spot at 4 and 14 days in the $\mathrm{MCAO}+\mathrm{CD} 200 \mathrm{R} \mathrm{Ab}$ group (group main effect, $F_{4,312}=37.42, P<0.0001$ ) (Fig. 4b), whereas it took less time to remove the spot at 7,14 , and 21 days in the $\mathrm{MCAO}+\mathrm{CD} 200 \mathrm{Fc}$ group compared with the MCAO+IgG group (group main effect, $F_{4,312}=44.91, P<0.0001$ ) (Fig. 4c). CD200Fc increased the frequency of injured forelimb use at 4 and 7 days after MCAO as determined by the limb-use asymmetry test (group main effect, $F_{4,292}=29.75, P<0.0001$ ) (Fig. 4d). In the modified grip-traction test, the time spent hanging onto the rope was increased markedly at 4, 7, 14, and 21 days after $\mathrm{MCAO}$ in the MCAO+CD200Fc group compared with the MCAO+IgG group. In contrast, CD200R Ab administration decreased the time spent hanging onto the rope at 4 days after MCAO (group main effect, $F_{4,364}=47.51$, $P<0.0001$ ) (Fig. 4e). We also detected the effect of CD200/CD200R signaling on the size of cerebral infarction. The result shown that CD200/CD200R signaling pathway modification did not influence the size of cerebral infarction at 28 days after MCAO (group main effect, $F_{4,30}=66.06, P<0.0001$ ) (Fig. 4f, g). These data suggest that activation of the CD200/CD200R signaling pathway promoted spontaneous functional recovery after stroke in rats.

Synaptic plasticity changes in cerebral ischemia rats after CD200/CD200R signaling pathway alteration

Neuronal connectivity and synaptic plasticity are essential to achieve complex tasks such as sensorimotor behavior. Thus, we sought to determine whether the CD200/CD200R signaling pathway participates in regulating synaptic plasticity after MCAO. Data from Western blotting demonstrated that the protein level of PSD-95 was increased significantly at 4, 7, and 28 days (group main effect, $F_{4,30}=101.1, P<0.0001$ ), and SYP levels were increased at 28 days (group main effect, $F_{4,30}=23.64, P<0.0001$ ) after CD200Fc i.c.v injection post-stroke (Fig. $5 \mathrm{a}-\mathrm{c}$ ). In contrast, the expression of SYP was reduced markedly at 4 days after CD200R Ab injection post-stroke (group main effect, $F_{4,30}=23.64, P<0.0001$ ) (Fig. 5a, c). Moreover, the stroke-induced reduction in spine density was rescued (group main effect, $F_{4,15}=36.91, P_{<} 0.0001$ ), and spine shape was altered from stubby to mushroom by CD200Fc application at 28 days after MCAO (group 

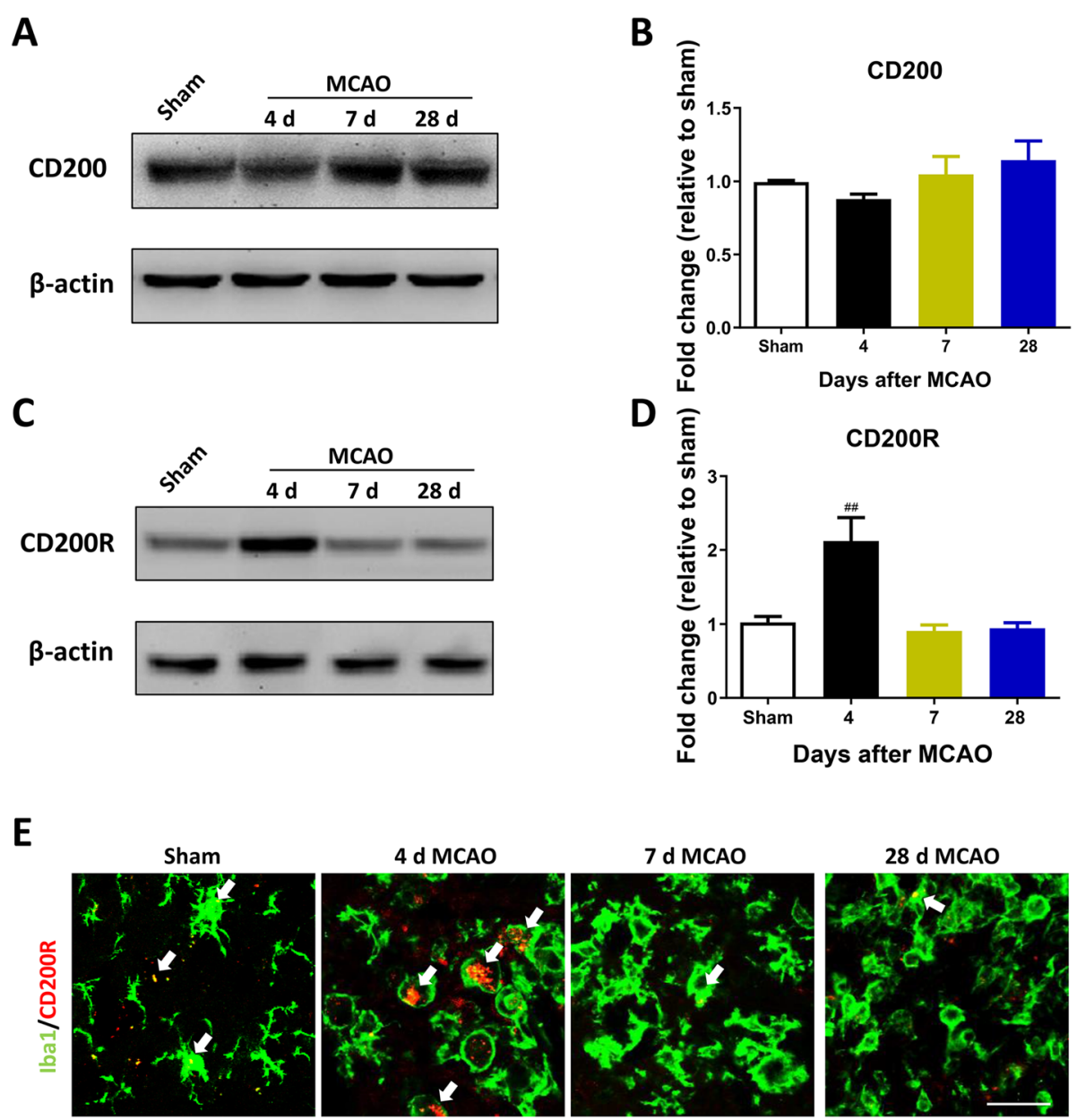

Fig. 3 CD200R was transiently upregulated after ischemic stroke in rats. Representative western blots of CD200, CD200R, and their corresponding normalized control $\beta$-actin in ipsilateral sensorimotor cortex at 4 days, 7 days, and 28 days after MCAO (a, $\mathbf{c})$. The levels of CD200R was upregulated at 4 days after MCAO, but MCAO did not influence the expression of CD200 at each time point (b, d). CD200R (red) staining showed protein expression at 4 days and colocalized with $\mathrm{Iba}^{+}$(green) cells (Iba1: a microglia marker) after MCAO in ipsilateral sensorimotor cortex (e) $(n=3$, Scale bar: $20 \mu \mathrm{m})$. Results are expressed as means \pm SEM. ${ }^{\# \# \#} P<0.001,{ }^{\# \#} P<0.01,{ }^{\#} P<0.05$ versus the Sham group

main effect, $F_{4,30}=0.5636, P=0.6909$ ) (Fig. $5 \mathrm{~d}-\mathrm{f}$ ). These results suggest that CD200Fc may enhance synaptic plasticity, thereby promoting sensorimotor functional recovery.

\section{Microglial response and inflammatory factor release after CD200/CD200R signaling pathway modification in} cerebral ischemia rats

Synaptic plasticity is impaired by exaggerated inflammatory responses and microglia activation during central nervous system (CNS) injury and neurodegenerative diseases $[11,31]$, and the CD200/CD200R signaling pathway plays an important role in regulating microglia activation and inflammatory factor release [32-34]. To investigate whether the CD200/CD200R signaling pathway is involved in regulating microglia activation and inflammatory factor release after stroke in rats, we assessed microglia activation using immunofluorescence and inflammatory factor (such as

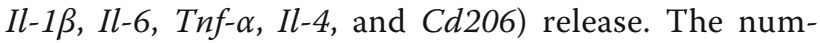
ber of $\mathrm{Iba}^{+} / \mathrm{CD} 68^{+}$cells in the ipsilateral sensorimotor cortex was decreased markedly at 4 and 7 days after MCAO in the MCAO+CD200Fc group compared with the MCAO+IgG group (group main effect, $F_{4,30}$ $=313.5, P<0.0001$ ) (Fig. 6a, b). In contrast, CD200R $\mathrm{Ab}$ significantly increased the number of Iba1+/ CD68+ cells at 4 and 7 days after MCAO (group main effect, $F_{4,30}=313.5, P<0.0001$ ) (Fig. $6 \mathrm{a}, \mathrm{b}$ ). The mRNA levels of $I l-1 \beta, I l-6, T n f-\alpha$, and $C d 206$ were downregulated, whereas $\mathrm{Il}-4$ was upregulated, following CD200Fc application after MCAO (Il-1 $\beta$ at 4 and 7 days after MCAO; Il- 6 and Tnf- $\alpha$ at 4 days after MCAO; $C d 206$ at 7 days after MCAO; $\mathrm{Il}-4$ at 7 days after MCAO) $(P<0.05)$ (Fig. $6 \mathrm{c}-\mathrm{g})$. In contrast, CD200R $\mathrm{Ab}$ increased the mRNA levels of $\mathrm{Il}-1 \beta$ at 4 and 7 days after MCAO and of $C d 206$ at 28 days $(P<0.05)$ 


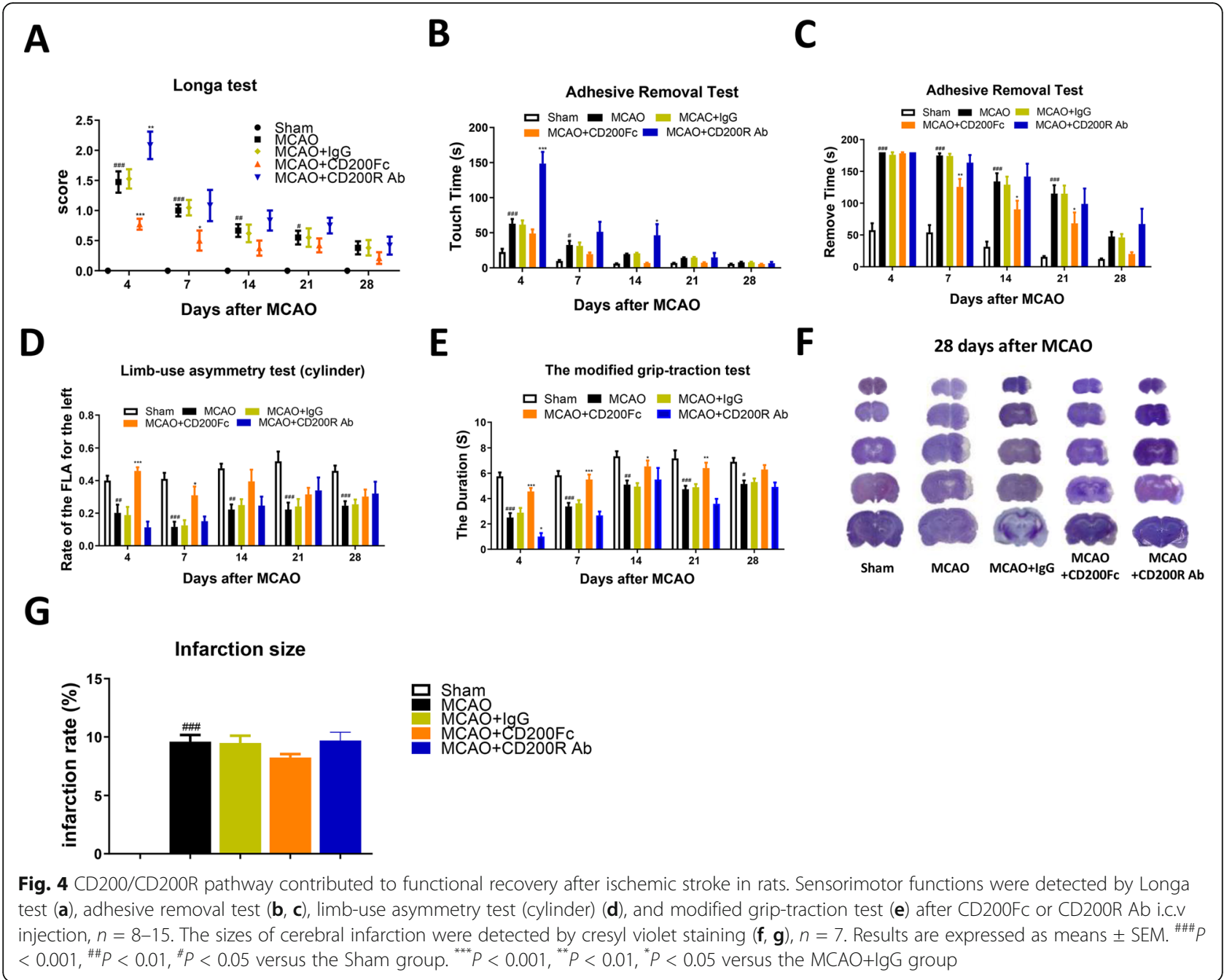

(Fig. 6a, g). These observations suggest that activation of the CD200/CD200R signaling pathway could ameliorate microglia activation and inflammatory factor release after stroke in rats.

\section{The effect of CD200/CD200R signaling pathway modification on NF- $K B$ and $p-M A P K$ expression after cerebral ischemia in rats}

Previous studies showed that activating the CD200/ CD200R signaling pathway limited microglia activation and inflammatory responses by inhibiting the $\mathrm{NF}-\kappa \mathrm{B}$ and mitogen-activated protein kinase (MAPK) pathways [35, 36]. To further investigate the mechanism through which CD200/CD200R signaling regulates synaptic plasticity after stroke, we assessed the expression of $\mathrm{p}$-p65, phospho-c-Jun N-terminal kinase (p-JNK), phospho-extracellular signal-regulated kinase$1 / 2$ (p-ERK1/2), and phospho-protein 38 (p-p38) MAPKs in the ipsilateral sensorimotor cortex. P65 phosphorylation was observed in the MCAO group at
7 and 28 days compared with the sham group (group main effect, $F_{4,30}=26.20, P<0.0001$ ) (Fig. 7a, b), but the CD200/CD200R signaling pathway did not influence p65 phosphorylation at each time point after MCAO. The protein levels of $\mathrm{p}-\mathrm{JNK}, \mathrm{p}-\mathrm{ERK}$, and $\mathrm{p}$ p38 were increased after MCAO compared with the sham group (p-JNK at 4, 7, and 28 days after MCAO, group main effect, $F_{4,30}=141.5, P<0.0001$; p-ERK at 4 days after MCAO, group main effect, $F_{4,30}=$ 10.64, $P<0.0001 ; \mathrm{p}-\mathrm{p} 38$ at 4 and 7 days after MCAO, group main effect, $F_{4,30}=36.43, P<0.0001$ ) (Fig. 7a, c-e). The protein levels of p-JNK and p-p38 were decreased significantly by CD200Fc (p-JNK at 4, 7, and 28 days after MCAO; p-p38 at 4 days after MCAO) (Fig. 7a, c, e). CD200R Ab increased the expression of p-JNK at 4 days and p-p38 at 4 and 7 days after MCAO compared with the $\mathrm{MCAO}+\mathrm{IgG}$ group (Fig. 7a, c, e). This suggests that activating the CD200/CD200R signaling pathway downregulates p-MAPK expression after stroke in rats. 


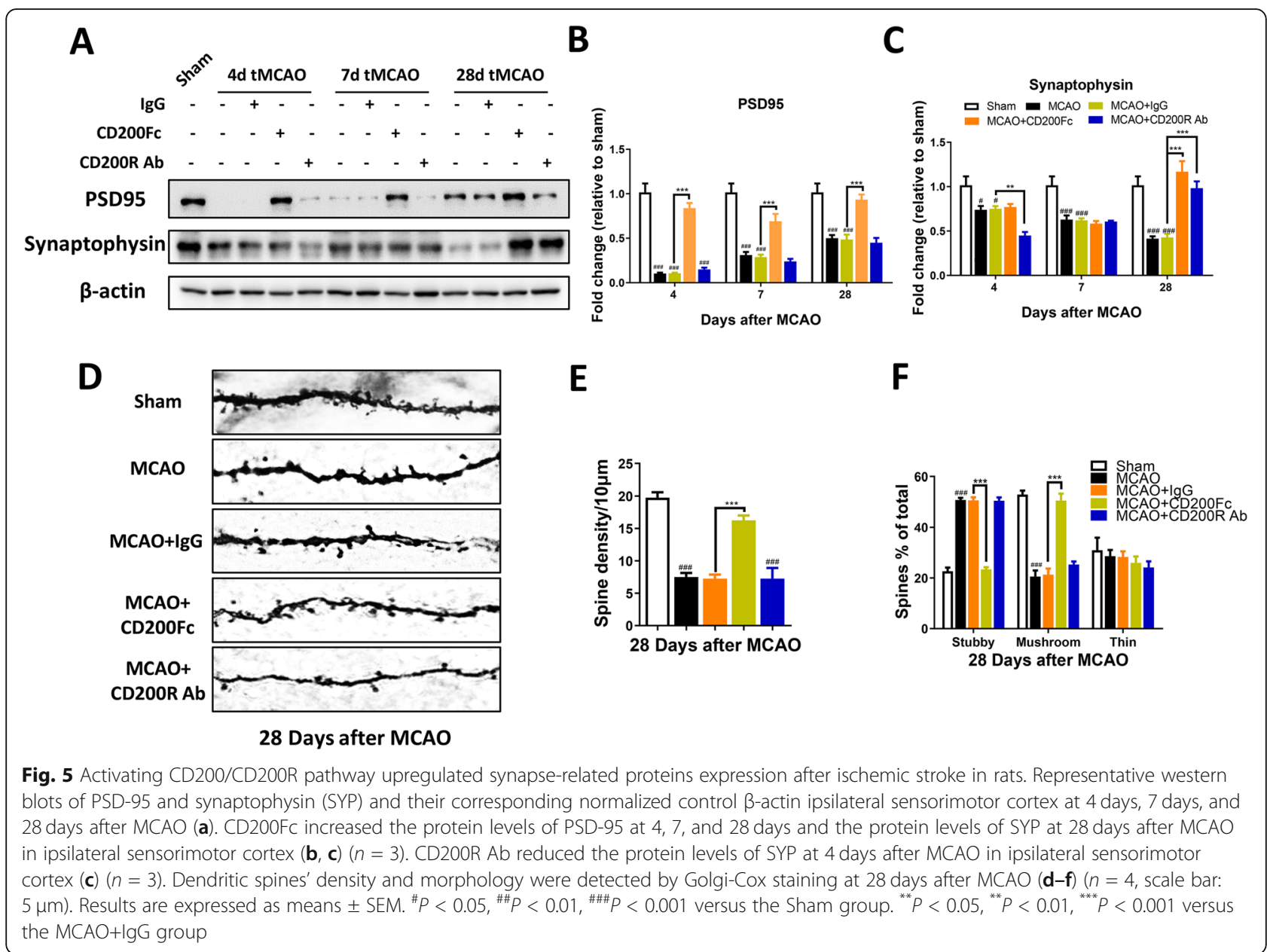

\section{CD200Fc inhibited JNK and P38 phosphorylation after OGD in BV2 microglia cell line}

To test whether CD200Fc inhibited microglial activation is mediated via MAPKs especially JNK and p38, we used BV2 cells to test the effect of CD200Fc on JNK and P38 phosphorylation after OGD. As shown in Fig. 8a-c, JNK and P38 phosphorylation induced by OGD were reversed by CD200Fc (p-JNK, $F$ 2, $6=0.009141, P=$ 0.9909; p-P38, $\left.F_{2,6}=1.289, P=0.3423\right)$. Next, we further determined the effect of CD200Fc on JNK and P38 phosphorylation in microglia via anisomycin (agonists of these JNK and P38), and the results showed that CD200Fc significantly decreased the protein level of $\mathrm{p}$ JNK and p-p38 induced by anisomycin (p-JNK, $F_{2,6}=$ 3.756, $P=0.0876$; p-P38, $F_{2,6}=5.044, P=0.0519$ ). These data indicated that CD200Fc could inhibit JNK and p38 phosphorylation in microglia.

\section{Discussion}

Recent studies indicated that the CD200/CD200R signaling pathway regulates the microglia activation-induced inflammatory response and synaptic plasticity in vitro and in vivo [32, 35, 37], but few studies have been performed in stroke. Here, we investigated CD200/CD200R as a potential target for promoting spontaneous recovery. The collected data support the viewpoint that sensorimotor deficiency was partially recovered by promoting synaptic plasticity. Activation of the CD200/CD200R signaling pathway further promoted functional recovery after stroke.

Ischemic stroke impairs the integrity of neural networks and intercellular signal transmission and causes sensorimotor deficits [6]. Surviving neural networks rewire and form new structural and functional circuits during spontaneous functional recovery [5]. PSD95 and SYP are crucial indicators of synaptic plasticity, which is involved in maturation of excitatory synapses and stabilization of synaptic contacts $[38,39]$. Dendritic spine density is an important index of structural and functional plasticity, which is associated with rewiring of neuronal circuits; enhanced plasticity can contribute to recovery of cortical function $[5,40]$. In the present study, the 


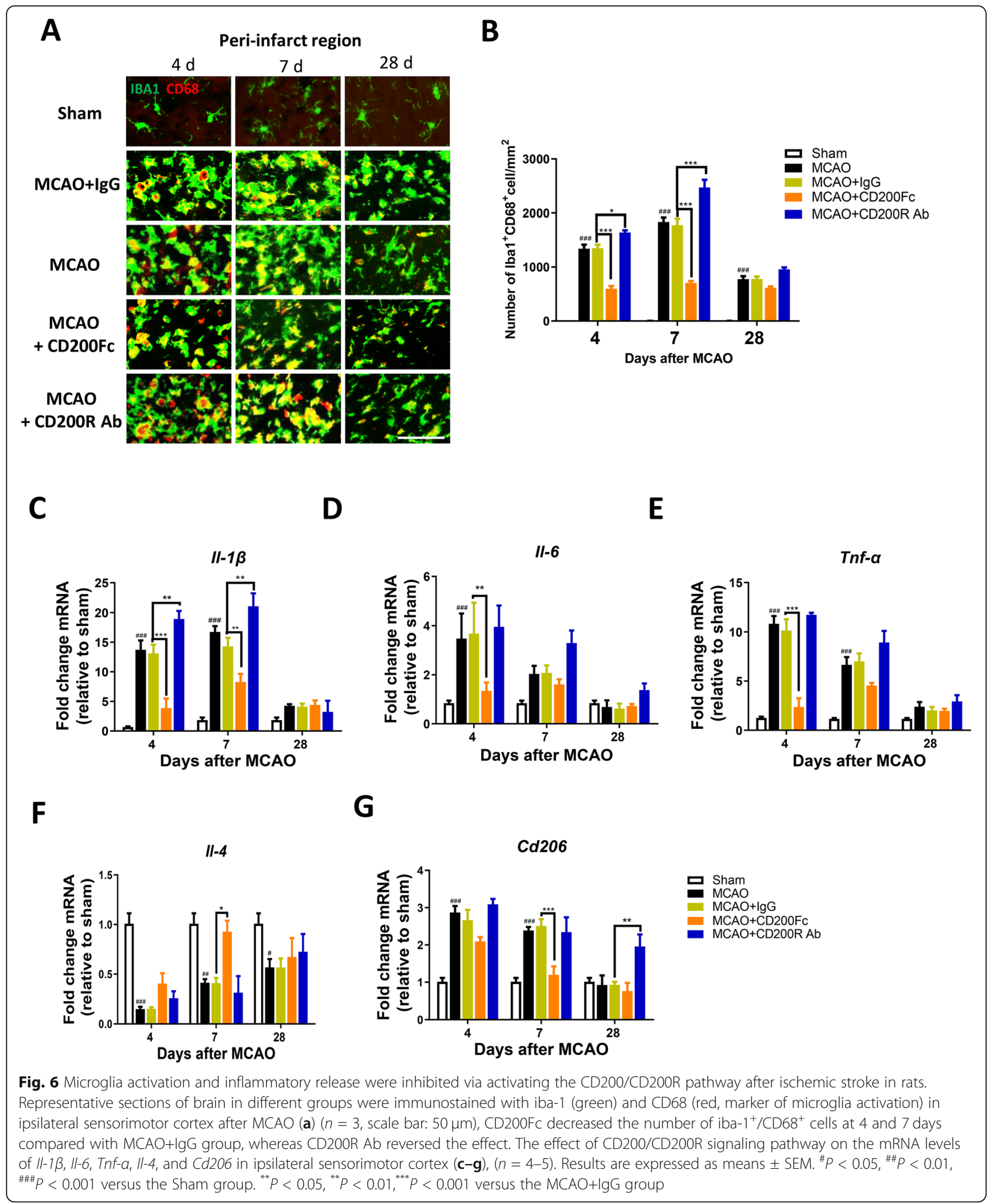

expression of PSD95 and dendritic spine density were increased significantly during spontaneous functional recovery, suggesting that neural networks were partially rewired after stroke.
The CD200/CD200R signaling pathway regulates synaptic plasticity, and dysfunction in this pathway contributes to synaptic deficits in aging and $A D[18$, 29]. In the current study, consistent with a previous 


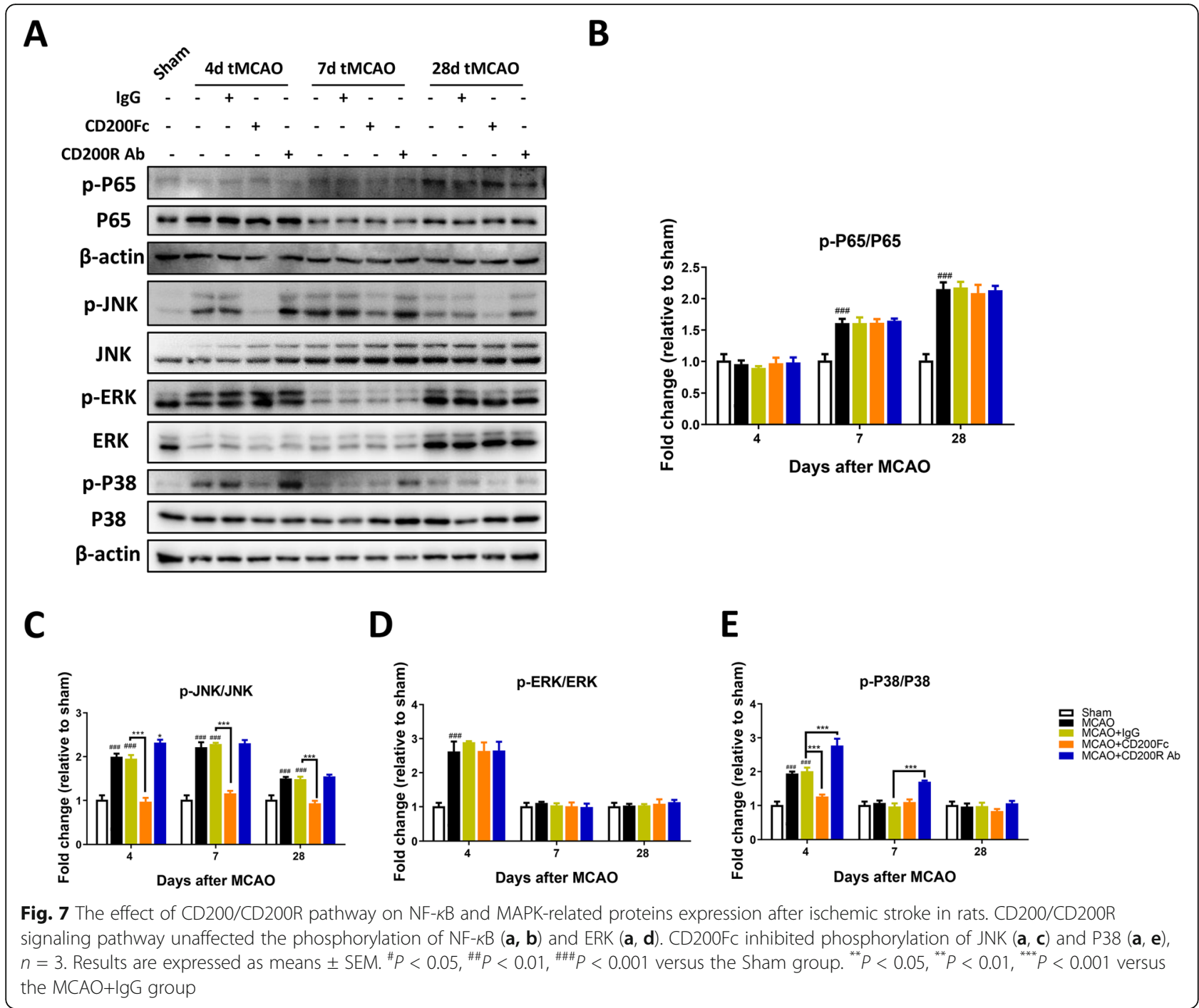

report [41], CD200R expression was transiently increased in microglia after stroke. Activating the CD200/CD200R signaling pathway with CD200Fc remarkably increased PSD95 expression and dendritic spine density. In addition, the sensorimotor functional dysfunction induced by stroke was ameliorated by CD200Fc application post-stroke. These observations showed that activation of the CD200/CD200R signaling pathway improved recovery of sensorimotor function by facilitating the formation of new neural networks and protecting synaptic structures from ischemia-induced damage.

Inflammation and pro-inflammatory factors are secondary injury mediators following cerebral ischemia [42], and they exacerbate loss of dendritic spines [11]. Suppressing microglia activation (microgliosis) rescues local inflammatory over-release and improves outcomes after stroke [43, 44]. The CD200/CD200R signaling pathway helps modulate microglia activation and inflammatory factors in many injury models [33, 41]. In this study, regulating CD200/CD200R signaling using CD200Fc significantly inhibited microglial over-activation and profoundly influenced the microenvironment, including decreasing the mRNA levels of proinflammatory (Il-1 1, Il-6, and Tnf- $\alpha$ ) factors and increasing the mRNA levels of anti-inflammatory (Il-4) regulators in the ipsilateral cortex. Similar effects were also observed after CD200Fc application in LPS-stimulated microglia cells, during aging, and in Parkinson's disease [18, 45]. These data suggest that CD200/CD200R signaling might help inhibit synapse loss after stroke by regulating the activation of microglia and modulating the balance between pro- and anti-inflammatory factors.

$\mathrm{NF}-\kappa \mathrm{B}$ is an important transcription factor that promotes microglia activation and inflammatory factor release $[46,47]$. Previous studies showed that CD200Fc could inhibit microglia activation and the inflammatory response by suppressing the NF- $\kappa \mathrm{B}$ pathway in vitro 


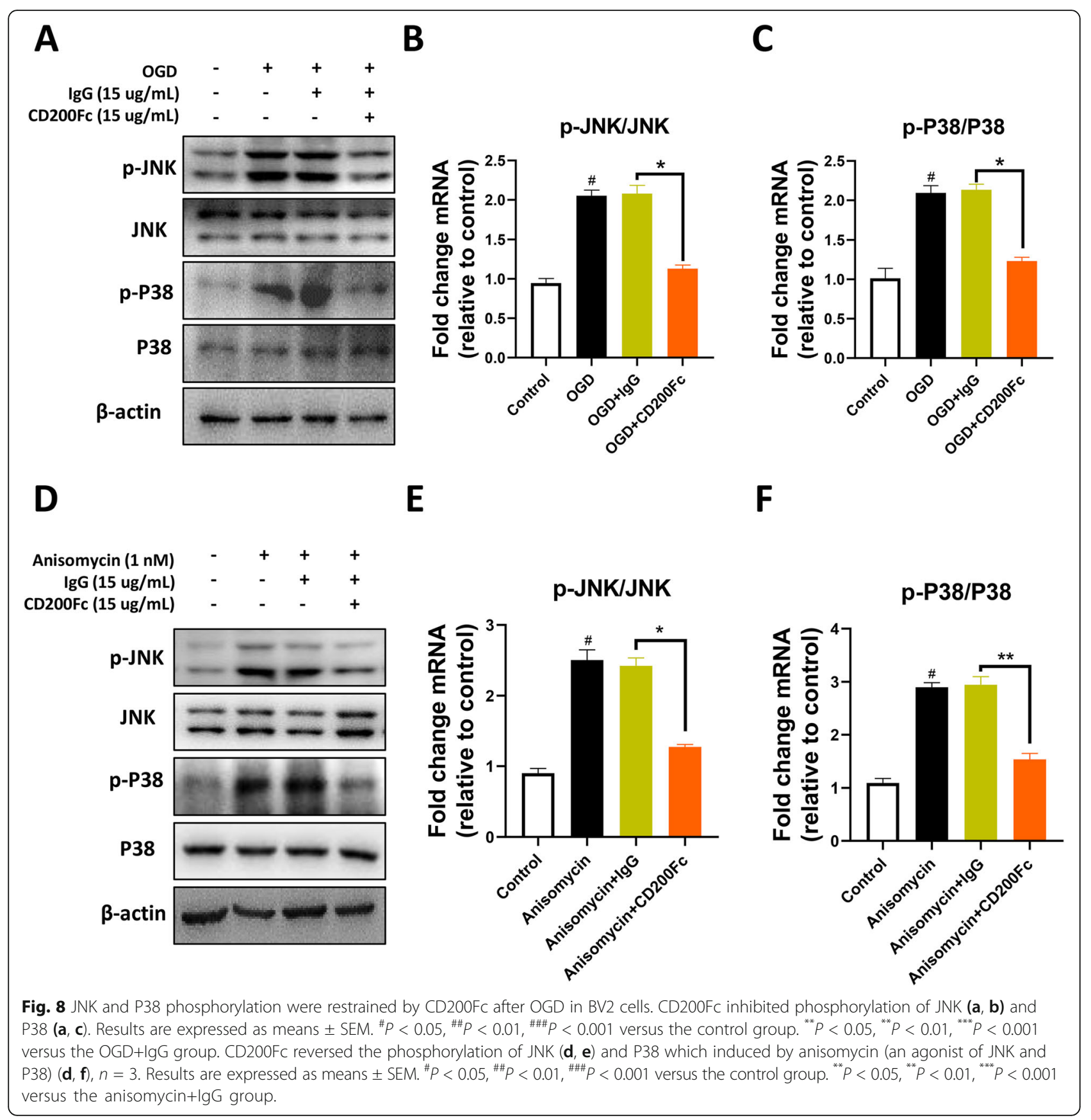

[48]. However, the current results showed that CD200/ CD200R signaling had no effect on the NF- $\kappa$ B pathway after stroke. CD200R is only expressed in microglia in the CNS, whereas inflammation is a complex response involving many kinds of cells. Activating CD200/ CD200R signaling may be insufficient to contain the NF$\kappa B$ pathway after stroke in vivo. Recent studies reported that the CD200/CD200R pathway modulated the inflammatory response by controlling MAPK activation in vitro and in vivo [49-51]. The current results showed that CD200Fc inhibited MAPK activation by suppressing the phosphorylation of p-JNK and p-p38 after stroke. This suggests that the effect of CD200/CD200R signaling on suppressing the inflammatory response may be exerted by inhibiting MAPK activation.

One limitation of the present study is that CD200R not only expressed in microglia, but also expressed in peripheral myeloid cells [52] and neuron in human according to the Ben Barres database. However, the infiltration of peripheral myeloid cells plays an important role in inflammatory response after stroke; CD200Fc or CD200R Ab may play a role in these cells. In this study, we did not 


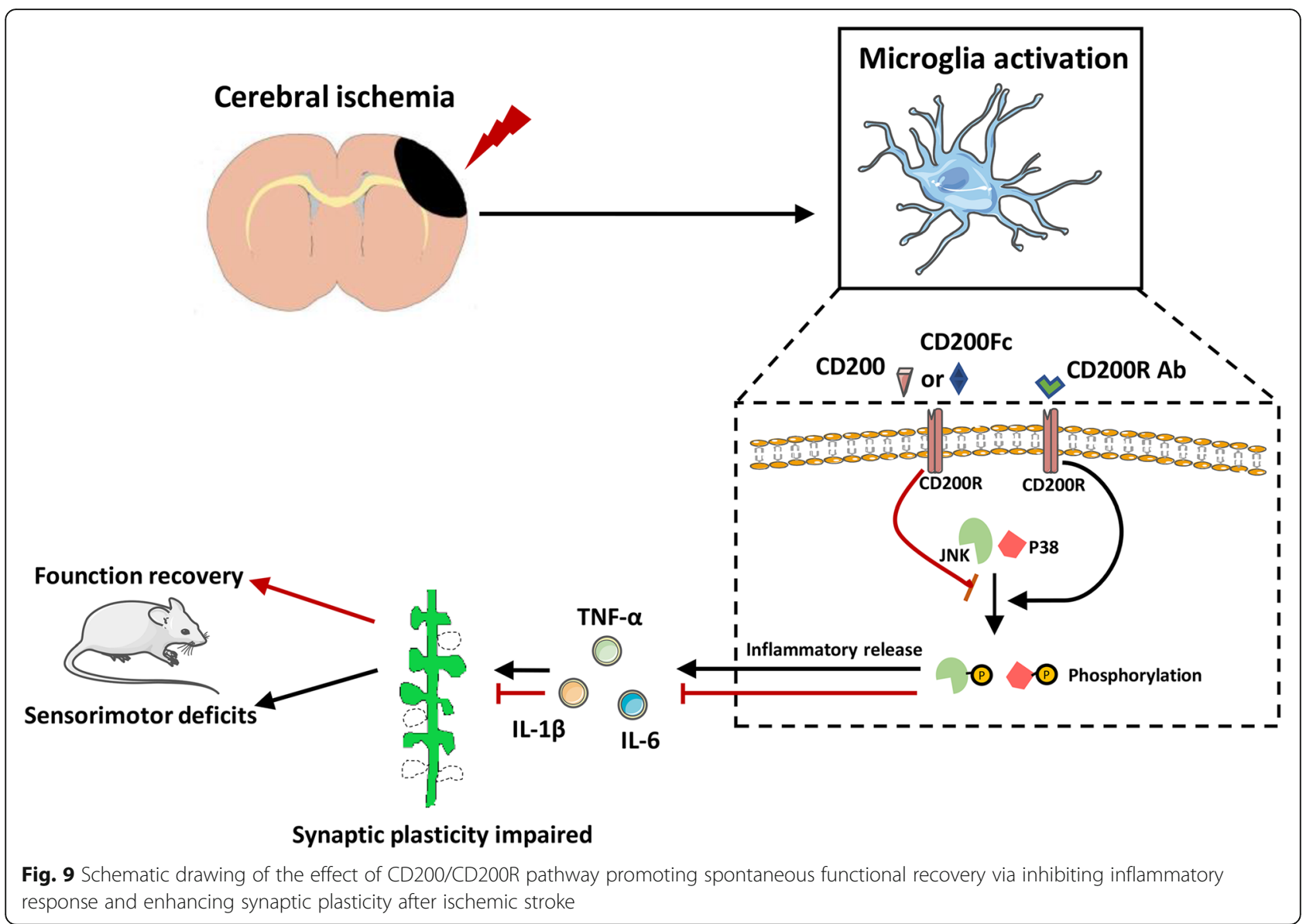

distinguish between microglia and infiltrating myeloid cells, and CD200R deficient on microglia transgenic mice would be helpful to explore the role of CD200/CD200R signaling on microglia after stroke in the future studies.

\section{Conclusion}

The CD200/CD200R signaling pathway participates in spontaneous functional recovery after stroke. Activation of CD200/CD200R signaling attenuated brain injury and facilitated the recovery of sensorimotor function. These effects were exerted by increasing synaptic plasticity via inhibiting microglia activation and improving the inflammatory microenvironment (Fig. 9). Therefore, the CD200/CD200R signaling pathway could be a potential therapeutic target for functional recovery after stroke.

\footnotetext{
Abbreviations

MCAO: Transient middle cerebral artery occlusion; CD200R: CD200 receptor; CD200R1: CD200 receptor1; CD200Fc: CD200 fusion protein; CD200R

Ab: CD200R blocking antibody; II-1 $\beta$ : Interleukin 1 beta; Tnf-a: Tumor necrosis factor alpha; II-6: Interleukin 6; II-4: Interleukin 4; Cd206: Cluster of differentiation 206; LTP: Long-term potentiation; CBF: Cerebral blood flow; PSD-95: Postsynaptic density protein 95; SYP: Synaptophysin; CNS: Central nervous system; Iba1: lonized calcium binding adapter molecule 1; NFKB: Nuclear factor kappa-B; MAPK: Mitogen-activated protein kinase; pJNK: Phospho-c-Jun N-terminal kinase; p-ERK1/2: Phospho-extracellular signalregulated kinase-1/2; OGD: Oxygen-glucose deprivation
}

Acknowledgements

Not applicable.

Authors' contributions

$H S, X H$, and $M H$ performed the most experiments. $H S, X T, T H$, and $M C$ contributed to the data analysis. HS wrote the manuscript. $\mathrm{HL}$ designed and supervised the study and reviewed the manuscript. All authors read and approved the final manuscript.

Funding

We gratefully acknowledge support from National Natural Science Foundation of China (81572240, 81772063), Natural Science Foundation of Jiang Su Province (BK20191325), and "Double First-Class" University project (CPU2018GY20, CPU2018GY13).

Availability of data and materials

The manuscript included all relevant data.

Ethics approval and consent to participate

All animal procedures were approved by the Animal Research Ethics Committee of China Pharmaceutical University (2019-11-004).

Consent for publication

Not applicable.

Competing interests

The authors declare that they have no competing interests. 


\section{Received: 22 November 2019 Accepted: 19 May 2020}

\section{Published online: 30 May 2020}

\section{References}

1. Ward NS. Restoring brain function after stroke - bridging the gap between animals and humans. Nat Rev Neurol. 2017;13:244-55.

2. Koh SH, Park HH. Neurogenesis in stroke recovery. Transl Stroke Res. 2017;8: 3-13.

3. Murphy $\mathrm{TH}$, Corbett D. Plasticity during stroke recovery: from synapse to behaviour. Nat Rev Neurosci. 2009;10:861-72.

4. Cassidy JM, Cramer SC. Spontaneous and therapeutic-induced mechanisms of functional recovery after stroke. Transl Stroke Res. 2017;8:33-46.

5. Murphy $\mathrm{TH}$, Corbett D. Plasticity during stroke recovery: from synapse to behaviour. Nature Reviews Neuroscience. 2009;10:861-72.

6. Sandvig I, Augestad IL, Haberg AK, Sandvig A. Neuroplasticity in stroke recovery. The role of microglia in engaging and modifying synapses and networks. European Journal of Neuroscience. 2018;47:1414-28.

7. Wang J, Pasinetti G. Epigenetic modulation of inflammation and synaptic plasticity promotes resilience against stress in mice. Abstr Pap Am Chem S. 2018;256:477.

8. Riazi K, Galic MA, Kentner AC, Reid AY, Sharkey KA, Pittman QJ. Microgliadependent alteration of glutamatergic synaptic transmission and plasticity in the hippocampus during peripheral inflammation. J Neurosci. 2015;35: 4942-52.

9. Rizzo FR, Musella A, De Vito F, Fresegna D, Bullitta S, Vanni V, Guadalupi L, Bassi MS, Buttari F, Mandolesi G, Centonze D, Gentile A. Tumor necrosis factor and interleukin-1 beta modulate synaptic plasticity during neuroinflammation. Neural Plast. 2018:1-2.

10. Qin C, Zhou LQ, Ma XT, Hu ZW, Yang S, Chen M, Bosco DB, Wu L, Tian DS. Dual functions of microglia in ischemic stroke. Neurosci Bull. 2019;35:921-33.

11. Ju FR, Ran YL, Zhu LR, Cheng XF, Gao H, Xi XX, Yang ZL, Zhang SX. Increased BBB permeability enhances activation of microglia and exacerbates loss of dendritic spines after transient global cerebral ischemia. Frontiers in Cellular Neuroscience. 2018;12:236.

12. Hoek RM, Ruuls SR, Murphy CA, Wright GJ, Goddard R, Zurawski SM, Blom B, Homola ME, Streit WJ, Brown MH, Barclay AN, Sedgwick JD. Downregulation of the macrophage lineage through interaction with OX2 (CD200). Science. 2000;290:1768-71.

13. Ren Y, Ye M, Chen SD, Ding JQ. CD200 Inhibits inflammatory response by promoting KATP channel opening in microglia cells in Parkinson's disease. Med Sci Monitor. 2016;22:1733-41.

14. Huang R, Lan QQ, Chen LF, Zhong HB, Cui L, Jiang L, Huang H, Li L, Zeng SM, Li M, Zhao X, Xu F. CD200Fc Attenuates retinal glial responses and RGCs apoptosis after optic nerve crush by modulating CD200/CD200R1 interaction. J Mol Neurosci. 2018;64:200-10.

15. Feng Z, Ye L, Klebe D, Ding Y, Guo ZN, Flores JJ, Yin C, Tang J, Zhang JH. Anti-inflammation conferred by stimulation of CD200R1 via Dok1 pathway in rat microglia after germinal matrix hemorrhage. J Cereb Blood Flow Metab. 2019;39:97-107

16. Costello DA, Lyons A, Denieffe S, Browne TC, Cox FF, Lynch MA. Long term potentiation is impaired in membrane glycoprotein CD200-deficient mice A ROLE FOR TOII-LIKE RECEPTOR ACTIVATION. J Biol Chem. 2011;286:34722-32.

17. Sharma N, Classen J, Cohen LG. Neural plasticity and its contribution to functional recovery. Handb Clin Neurol. 2013;110:3-12.

18. Cox FF, Carney D, Miller AM, Lynch MA. CD200 fusion protein decreases microglial activation in the hippocampus of aged rats. Brain Behav Immun. 2012;26:789-96.

19. Feng DP, Huang AQ, Yan W, Chen DZ. CD200 dysfunction in neuron contributes to synaptic deficits and cognitive impairment. Biochem Bioph Res Co. 2019:516:1053-9.

20. Longa EZ, Weinstein PR, Carlson S, Cummins R. Reversible middle cerebral artery occlusion without craniectomy in rats. Stroke; a journal of cerebral circulation. 1989;20:84.

21. Liguz-Lecznar M, Zakrzewska R, Daniszewska K, Kossut M. Functional assessment of sensory functions after photothrombotic stroke in the barrel field of mice. Behav Brain Res. 2014;261:202-9.

22. MacLellan CL, Auriat AM, McGie SC, Yan RH, Huynh HD, De Butte MF, Colbourne F. Gauging recovery after hemorrhagic stroke in rats: implications for cytoprotection studies. J Cereb Blood Flow Metab. 2006;26:1031-42.
23. Liu Y, Li CH, Wang J, Fang YQ, Sun H, Tao X, Zhou XF, Liao H. Nafamostat mesilate improves neurological outcome and axonal regeneration after stroke in rats. Mol Neurobiol. 2017;54:4217-31.

24. De Geyter D, Stoop W, Sarre S, De Keyser J, Kooijman R. Neuroprotective efficacy of subcutaneous insulin-like growth factor-I administration in normotensive and hypertensive rats with an ischemic stroke. Neuroscience. 2013;250:253-62.

25. Cao LJ, Chen JY, Li M, Qin YY, Sun ML, Sheng R, Han F, Wang GH, Qin ZH. Endogenous level of TIGAR in brain is associated with vulnerability of neurons to ischemic injury. Neurosci Bull. 2015;31:527-40.

26. Su F, Guo AC, Li WW, Zhao YL, Qu ZY, Wang YJ, Wang Q, Zhu YL. Low-dose ethanol preconditioning protects against oxygen-glucose deprivation/ reoxygenation-induced neuronal injury by activating large conductance, Ca2+-activated K+ channels in vitro. Neurosci Bull. 2017;33:28-40.

27. Esposito E, Hayakawa K, Maki T, Arai K, Lo EH. Effects of postconditioning on neurogenesis and angiogenesis during the recovery phase after focal cerebral ischemia. Stroke. 2015;46:2691-4.

28. Di Pino G, Pellegrino G, Assenza G, Capone F, Ferreri F, Formica D, Ranieri F, Tombini M, Ziemann U, Rothwell JC, Di Lazzaro V. Modulation of brain plasticity in stroke: a novel model for neurorehabilitation. Nat Rev Neurol. 2014;10:597-608.

29. Feng D, Huang A, Yan W, Chen D. CD200 dysfunction in neuron contributes to synaptic deficits and cognitive impairment. Biochem Biophys Res Commun. 2019;516:1053-9.

30. Ojo B, Rezaie P, Gabbott PL, Davies H, Colyer F, Cowley TR, Lync M, Stewart MG. Age-related changes in the hippocampus (loss of synaptophysin and glial-synaptic interaction) are modified by systemic treatment with an NCAM-derived peptide. FGL, Brain Behav Immun. 2012;26:778-88.

31. Norden DM, Muccigrosso MM, Godbout JP. Microglial priming and enhanced reactivity to secondary insult in aging, and traumatic CNS injury, and neurodegenerative disease. Neuropharmacology. 2015;96:29-41.

32. Manich G, Recasens M, Valente T, Almolda B, Gonzalez B, Castellano B. Role of the CD200-CD200R axis during homeostasis and neuroinflammation. Neuroscience. 2019:405:118-36.

33. Lago N, Pannunzio B, Amo-Aparicio J, Lopez-Vales R, Peluffo H. CD200 modulates spinal cord injury neuroinflammation and outcome through CD200R1. Brain Behav Immun. 2018;73:416-26.

34. Zhao X, Li J, Sun HT. CD200-CD200R Interaction: An Important Regulator After Stroke. Front Neurosci-Switz. 2019;13:840.

35. Jiang L, Xu F, He W, Chen L, Zhong H, Wu Y, Zeng S, Li L, Li M. CD200FC reduces TLR4-mediated inflammatory responses in LPS-induced rat primary microglial cells via inhibition of the NF-kappaB pathway. Inflamm Res. 2016; 65:521-32.

36. Zhang SL, Cherwinski H, Sedgwick JD, Phillips JH. Molecular mechanisms of CD200 inhibition of mast cell activation. J Immunol. 2004;173:6786-93.

37. Liu C, Shen YF, Tang Y, Gu YC. The role of N-glycosylation of CD200CD200R1 interaction in classical microglial activation. J Inflamm-Lond. 2018; 15:28.

38. El-Hussein AE, Schnell E, Chetkovich DM, Nicoll RA, Bredt DS. PSD-95 involvement in maturation of excitatory synapses. Science. 2000;290:1364-8.

39. Taft CE, Turrigiano GG. PSD-95 promotes the stabilization of young synaptic contacts. Philos T R Soc B. 2014;369:20130134.

40. Ward NS, Cohen LG. Mechanisms underlying recovery of motor function after stroke. Arch Neurol. 2004;61:1844-8.

41. Ritzel RM, Al Mamun A, Crapser J, Verma R, Patel AR, Knight BE, Harris N, Mancini N, Roy-O'Reilly M, Ganesh BP, Liu FD, McCullough LD. CD200CD200R1 inhibitory signaling prevents spontaneous bacterial infection and promotes resolution of neuroinflammation and recovery after stroke. J Neuroinflamm. 2019;16:40.

42. Kim JY, Kawabori M, Yenari MA. Innate inflammatory responses in stroke: mechanisms and potential therapeutic targets. Curr Med Chem. 2014;21: 2076-97.

43. Walter HL, van der Maten $G$, Antunes AR, Wieloch T, Ruscher K. Treatment with AMD3100 attenuates the microglial response and improves outcome after experimental stroke. J Neuroinflamm. 2015;12:24.

44. Kauppinen TM, Suh SW, Berman AE, Hamby AM, Swanson RA. Inhibition of poly (ADP-ribose) polymerase suppresses inflammation and promotes recovery after ischemic injury. J Cerebr Blood F Met. 2009;29:820-9.

45. Zhang S, Wang XJ, Tian LP, Pan J, Lu GQ, Zhang YJ, Ding JQ, Chen SD. CD200-CD200R dysfunction exacerbates microglial activation and 
dopaminergic neurodegeneration in a rat model of Parkinson's disease. J Neuroinflamm. 2011;8:154.

46. Shabab T, Khanabdali R, Moghadamtousi SZ, Kadir HA, Mohan G. Neuroinflammation pathways: a general review. Int J Neurosci. 2017;127: 624-33.

47. Harari OA, Liao JK. NF-kappaB and innate immunity in ischemic stroke. Ann N Y Acad Sci. 2010;1207:32-40.

48. Jiang L, Xu F, He WJ, Chen LF, Zhong HB, Wu Y, Zeng SM, Li L, Li M. CD200Fc reduces TLR4-mediated inflammatory responses in LPS-induced rat primary microglial cells via inhibition of the NF-kappa B pathway. Inflammation Research. 2016;65:521-32.

49. Zhang S, Cherwinski H, Sedgwick JD, Phillips JH. Molecular mechanisms of CD200 inhibition of mast cell activation. J Immunol. 2004;173:6786-93.

50. Wang XJ, Zhang S, Yan ZQ, Zhao YX, Zhou HY, Wang Y, Lu GQ, Zhang JD. Impaired CD200-CD200R-mediated microglia silencing enhances midbrain dopaminergic neurodegeneration: roles of aging, superoxide, NADPH oxidase, and p38 MAPK. Free Radic Biol Med. 2011;50:1094-106.

51. B. Zhu, Y. Yu, X. Liu, Q. Han, Y. Kang, L. Shi, CD200 Modulates S. aureusinduced innate immune responses through suppressing p38 signaling, Int J Mol Sci, 20 (2019) 659.

52. Darmochwal-Kolarz DA, Kludka-Sternik M, Chmielewski T, Kolarz B, Rolinski J, Leszczynska-Gorzelak B, Oleszczuk J. The expressions of CD200 and CD200R molecules on myeloid and lymphoid dendritic cells in pre-eclampsia and normal pregnancy. Am J Reprod Immunol. 2012;67:474-81.

\section{Publisher's Note}

Springer Nature remains neutral with regard to jurisdictional claims in published maps and institutional affiliations.

Ready to submit your research? Choose BMC and benefit from:

- fast, convenient online submission

- thorough peer review by experienced researchers in your field

- rapid publication on acceptance

- support for research data, including large and complex data types

- gold Open Access which fosters wider collaboration and increased citations

- maximum visibility for your research: over $100 \mathrm{M}$ website views per year

At $\mathrm{BMC}$, research is always in progress.

Learn more biomedcentral.com/submissions 